Geometry 8 Topology Monographs

Volume 2: Proceedings of the Kirbyfest

Pages 87-102

\title{
Topological Field Theories and formulae of Casson and Meng-Taubes
}

\author{
S K DONALDSON
}

\begin{abstract}
The goal of this paper is to give a new proof of a theorem of Meng and Taubes [9] that identifies the Seiberg-Witten invariants of 3-manifolds with Milnor torsion. The point of view here will be that of topological quantum field theory. In particular, we relate the SeibergWitten equations on a 3-manifold with the Abelian vortex equations on a Riemann surface. These techniques also give a new proof of the surgery formula for the Casson invariant, interpreted as an invariant of a homology $S^{2} \times S^{1}$.
\end{abstract}

AMS Classification 57R57; 57M25, 57N10, 58D29

Keywords Seiberg-Witten invariant, Casson invariant, Alexander polynomial, Milnor torision, topological quantum field theory, moduli space, vortex equation

\section{Introduction}

In 1985, Casson introduced his renowned invariant of homology 3-spheres, together with a surgery formula for the change in the invariant when the manifold is changed by a surgery [1]. The latter can also be seen as a formula for a Casson-type invariant of a homology $S^{1} \times S^{2}$. For a manifold $Y^{3}$ of this kind we "count", in the sense of Fredholm differential topology - as in the work of Taubes [11] - the flat connections on a non-trivial $S O(3)$ bundle over $Y$ to obtain a number $C(Y)$. Casson's formula is then

$$
C(Y)=\sum_{i>0} i^{2} a_{i}
$$

where the integers $a_{i}$ are the coefficients of the normalised Alexander polynomial $\Delta_{Y}(t)=a_{0}+\sum_{i>0} a_{i}\left(t^{i}+t^{-i}\right)$. (The interpretation of Casson's surgery formula in this way is implicit in the Floer's exact sequence [4] relating the Floer homologies of manifolds differing by surgeries.)

In 1995, Meng and Taubes [9] considered the dimensionally-reduced SeibergWitten equations over homology $S^{1} \times S^{2}$ 's. Here one has an integer parameter 
$d>0$ and invariants $S W_{d}(Y)$ which count the solutions of the equations on a complex line bundle of Chern class $d$ over $Y$. The Meng-Taubes formula can be written

$$
S W_{d}(Y)=a_{d+1}+2 a_{d+2}+3 a_{d+3}+\ldots
$$

The purpose of this article is to describe how the ideas of Topological Field Theory can be applied to derive these formulae in ways that are, at least superficially, different from the original proofs. The author has developed this point of view intermittently over the last 10 years. The Casson case was worked out in discussions with M Furuta around 1989, partly inspired by lectures of Segal on Quantum Field Theory (and some of the constructions described in Section 5 are due to Furuta). The Seiberg-Witten case was worked out in 1995, partly motivated by the programme of Pidstragatch and Tyurin and work of Thaddeus, which we mention again at the end of the article. Over the years a number of authors have developed ideas which come very close to the point of view we take here: we mention particularly the work of Frohman and Nicas [5] on the one hand and Hutchings and Lee [7] on the other. But we hope that the approach here may add something which does not quite appear in the literature and which may be worth recording; without staking any particular claims to originality. To keep the exposition short and simple we take the liberty of treating the differential-geometric and analytical foundations of our arguments - which we believe can all be pieced together from the literature - very sketchily, and concentrate on the formal aspects. (As a particular instance of this sketchiness, we will not go into the question of orientations, so our formulae will really be derived up to overall sign \pm 1 .)

\section{Topological field theories}

We will consider theories which satisfy the Topological Field Theory axioms [2] in a restricted range of cases. In essence we want to associate to each closed, connected, oriented surface $\Sigma$ a vector space $V(\Sigma)$ and to a cobordism $W$ between two such $-\Sigma_{0}, \Sigma_{1}$-a linear map

$$
\rho_{W}: V\left(\Sigma_{0}\right) \rightarrow V\left(\Sigma_{1}\right),
$$

satisfying the familiar composition rule when one glues together two cobordisms. In our examples the spaces will come with natural gradings, although we will need only keep track of the $\mathbf{Z} / 2$-grading. In the case when $\Sigma_{0}, \Sigma_{1}$ are diffeomorphic, and we choose a definite diffeomorphism between them, we may 
form a closed 3-manifold $\bar{W}$. We consider theories where we have numerical invariants for such closed manifolds $n(\bar{W})$ and the other gluing axiom we require is that

$$
n(\bar{W})=\operatorname{Tr}_{s}\left(\rho_{W}\right),
$$

where $\rho_{W}$ is regarded as an endomorphism of $V\left(\Sigma_{0}\right)$, using the fixed identification of $\Sigma_{0}, \Sigma_{1}$ and $\operatorname{Tr}_{s}$ denotes a "supertrace", with signs induced by the grading.

We will now recall how formal structures of this kind arise in gauge theory. We begin with the set-up which will correspond to the Casson formula. For each surface $\Sigma$ we construct the moduli space $M(\Sigma)$ of flat connections on a non-trivial $S O(3)$ bundle over $\Sigma$. This is an orbifold of dimension $6 g(\Sigma)-6$. Our vector space $V^{C}(\Sigma)$ will then be the homology of this moduli space. A technical variant of this construction is to consider the moduli space of projectively flat connections on a $U(2)$ bundle of odd-degree over $\Sigma$, with fixed central curvature. The moduli space of these connections is a smooth manifold $\tilde{M}(\Sigma)$ covering (in the orbifold sense) $M(\Sigma)$. More precisely, $M(\Sigma)$ is the quotient of $\tilde{M}(\Sigma)$ by a natural action of $H^{1}(\Sigma ; \mathbf{Z} / 2)$. It is somewhat easier to work in this $U(2)$ framework, since one does not run into the notorious difficulties with reducible connections, but the language becomes more tortuous. In the end it does not really matter how one proceeds since we will only be concerned with the real homology and it is known that the real homology groups of $M(\Sigma), \tilde{M}(\Sigma)$ are isomorphic. Equivalently the action of $H^{1}(\Sigma ; \mathbf{Z} / 2)$ on the real homology is trivial [3]. Likewise, we know that Poincaré duality and intersection theory goes over for the real homology of an orbifold. To simplify our exposition we will work with the $S O(3)$ moduli spaces and ignore the fact that they are not quite manifolds.

Turning to 3-manifolds, for a closed, oriented, 3-manifold $Y$ with the homology of $S^{1} \times S^{2}$ we consider of course the invariant $C(Y)$ obtained by counting the flat $S O(3)$ connections on a non-trivial bundle. Note that the topological hypotheses mean that there is a unique non-trivial $S O(3)$ bundle over $Y$, and there are no reducible flat connections on this bundle. We could extend the theory to certain other 3-manifolds, but we would need to specify the relevant bundle and avoid reducibles, so for simplicity we will stick to the class of homology $S^{1} \times S^{2}$ 's. Now let $W$ be a cobordism from $\Sigma_{0}$ to $\Sigma_{1}$. We will restrict attention to the cobordisms $W$ such that $H_{1}\left(\Sigma_{0}\right) \oplus H_{1}\left(\Sigma_{1}\right)$ maps onto $H_{1}(W)$ under the homorphisms induced by inclusion, or equivalently that

$$
H_{1}(W, \partial W)=\mathbf{Z} .
$$

All the manifolds we encounter - occurring the decomposition of a homology $S^{1} \times S^{2}$ —will satisfy this condition. The main idea is this: we consider a 
moduli space $M(W)$ of flat connections over $W$, with a restriction map

$$
r: M(W) \rightarrow M\left(\Sigma_{0}\right) \times M\left(\Sigma_{1}\right) .
$$

If $M(W)$ is a compact, oriented manifold it carries a fundamental homology class which can be pushed forward to $r_{*}[M(W)] \in H_{*}\left(M\left(\Sigma_{0} \times M\left(\Sigma_{1}\right)\right.\right.$. Then Poincare duality and the Kunneth theorem give an identification

$$
\begin{aligned}
H_{*}\left(M\left(\Sigma_{0}\right) \times M\left(\Sigma_{1}\right)\right) & \cong \operatorname{Hom}\left(H _ { * } \left(M\left(\Sigma_{0}\right), H_{*}\left(M\left(\Sigma_{1}\right)\right)\right.\right. \\
& =\operatorname{Hom}\left(V^{C}\left(\Sigma_{0}\right), V^{C}\left(\Sigma_{1}\right)\right),
\end{aligned}
$$

and we define $\rho_{W}$ to be map obtained in this way from $r_{*}[M(W)]$. Of course all of this needs to be interpreted with the usual caveats of the subject: we need to have a framework in which the equations defining the moduli space fit into a "Fredholm package", with a well-defined index or formal dimension, and we may need to perturb the equations to obtain transversality. There are two ways in which this can be done. In one approach, as in [11], one sets up an elliptic boundary-value problem on the manifold-with-boundary $W$. In the other one adds semi-infinite cylinders to the ends to construct a complete manifold $\hat{W}$. Then the set-up can be seen as a dimension-reduced version of the $(3+1)$-dimensional Floer theory, regarding flat connections over $\hat{W}$ as instantons over $\hat{W} \times S^{1}$. In either case one finds that the relevant index or formal dimension is $3 \chi(\partial W)$. The functorial property for compositions of cobordisms, and the relation with the numerical invariant $C(\bar{W})$ follow from the usual "gluing theory" arguments. Notice that any homology- $S^{1} \times S^{2}$ can be realised as a manifold $\bar{W}$, where $W$ satisfies (3).

The Seiberg-Witten case follows a similar pattern. Here it seems that one has to work in the tubular-end framework. We choose a Riemannian metric and spin structure on the manifold $\hat{W}$ and a line bundle $L \rightarrow \hat{W}$ with

$$
\left\langle c_{1}(L),\left[\Sigma_{0}\right]\right\rangle=\left\langle c_{1}(L), \Sigma_{1}\right\rangle=d .
$$

Then we have Seiberg-Witten equations for a pair consisting of a connection and an $L$-valued spinor field over $\hat{W}$. If $d>0$ we do not run into difficulties with reducible solutions. To find the appropriate moduli spaces associated with the boundary components we look at the translation-invariant solutions of these equations, which are the potential limits of finite-energy solutions over the cylindrical ends. These translation-invariant solutions over a tube $\Sigma \times \mathbf{R}$ are the solutions of a vortex equation over $\Sigma,[8]$. That is we fix a spin structure and metric on $\Sigma$ and look for pairs $(a, \psi)$ consisting of a connection $a$ on $L \rightarrow \Sigma$ and a section $\psi$ of $L^{*} \otimes S^{+}(\Sigma)$ such that

$$
\begin{aligned}
D_{a} \psi & =0 \\
* F(a) & =|\psi|^{2} .
\end{aligned}
$$


Recall that over the surface $\Sigma$ the spinor bundle $S^{+}$can be identified with the square root $K_{\Sigma}^{1 / 2}$ and the Dirac operator $D_{a}$ can be identified with the CauchyRiemann operator, so that $\psi$ becomes a holomorphic section of a holomorphic line bundle $L^{*} \otimes K^{1 / 2}$ of degree $k=g-1-d$. Thus the zero set of $\psi$ is a positive divisor of degree $k$, or equivalently an element of the symmetric product Sym ${ }^{k}(\Sigma)$. The basic result we need is that this sets up a 1-1 correspondence between the moduli space of solutions of the vortex equation and the symmetric product, provided $d>0$. (This is interpreted as saying that the moduli space is empty if $d>g-1$.) We see then that the vector space $V_{d}(\Sigma)$ we should associate to $\Sigma$ in this theory, for a given parameter $d$, is the real homology $H_{*}\left(\operatorname{Sym}^{k}(\Sigma)\right)$, where $k=g-1-d$. With this said, the general construction goes through much as before, appealing to the literature on the Seiberg-WittenFloer theory for the relevant gluing and transversality results.

\section{Homology of the moduli spaces}

An important part of the general topological field theory package is the fact that the vector space $V(\Sigma)$ carries a natural representation of the mapping class group of the surface $\Sigma$. (More precisely, in the Seiberg-Witten case we should consider finite-index subgroups of diffeomorphisms which preserve a spin structure.) The essence of our approach to the 3 -dimensional invariants is to obtain a good understanding of these representations. We do the SeibergWitten case first.

\section{(i) Homology of symmetric products}

For any space $X$ the real (or rational) homology of the symmetric products Sym ${ }^{k}(X)$ is easy to describe. We begin with the homology of the $k$-fold product $X \times \cdots \times X$, which is just the tensor product of $k$ copies of $H_{*}(X)$, and then take the invariant part under the action of the permutation group. In the case of a surface this gives

$$
H_{*}\left(\operatorname{Sym}^{k}(\Sigma)\right)=\left(\Lambda^{k} \otimes s^{0}\right) \oplus\left(\Lambda^{k-1} \otimes s^{1}\right) \oplus \cdots \oplus s^{k},
$$

where $\Lambda^{j}=\Lambda^{j}\left(H_{1}(\Sigma)\right)$ and $s^{j}$ is the jth. symmetric power of $H_{0}(\Sigma) \oplus H_{2}(\Sigma)$. So if we write $u$ for the fundamental class of $\Sigma$,

$$
s^{j}=\left\langle 1, u, \ldots, u^{j}\right\rangle,
$$

and $\operatorname{dim}\left(s^{j}\right)=j+1$.

It will be convenient to reorganise the grading of our exterior powers by writing

$$
\Lambda_{(i)}=\Lambda^{g-i}
$$


so we have isomorphisms $\Lambda_{(i)} \cong \Lambda_{(-i)}$ induced by the intersection form on $H_{1}(\Sigma)$. Thus, remembering that $k=g-1-d$, our formula (4) becomes

$$
V_{d}(\Sigma)=\Lambda_{(d+1)} \oplus\langle 1, u\rangle \otimes \Lambda_{(d+2)} \oplus\left\langle 1, u, u^{2}\right\rangle \otimes \Lambda_{(d+3)} \cdots,
$$

or, in shorthand,

$$
V_{d}(\Sigma)=\Lambda_{(d+1)}+2 \Lambda_{(d+2)}+3 \Lambda_{(d+3)} \cdots
$$

Here the reader will immediately see the shape of the Meng-Taubes formula (2) appearing. Tracing through the constructions one verifies easily enough that these formulae describe the vector spaces $V_{d}(\Sigma)$ as representations of the mapping class group. Notice that (although we will not need this) we can also bring in the grading of the homology groups of the symmetric products. Again it is best to reorganise the standard grading by writing:

$$
V_{d}^{(i)}(\Sigma)=H_{k-i}\left(\operatorname{Sym}^{k}(\Sigma)\right)
$$

so $V_{d}^{(0)}$ is the middle-dimensional homology. A convenient notation is then to write

$$
\sum V_{d}^{(i)}(\Sigma) t^{i}=\Lambda_{(d+1)}+\left(t+t^{-1}\right) \Lambda_{(d+2)}+\left(t^{2}+1+t^{-2}\right) \Lambda_{(d+3)}+\ldots,
$$

where we mean that the vector space appearing as the coefficients of $t^{i}$ on the left hand side is isomorphic to the corresponding direct sum on the right hand side.

\section{(ii) Homology of moduli spaces of flat connections}

We begin with the formulae, obtained by Atiyah and Bott and other authors using a variety of methods, for the Betti numbers of the moduli space $M(\Sigma)$. The Poincaré polynomial is

$$
\begin{aligned}
P_{t}(M(\Sigma)) & =\sum \operatorname{dim}\left(H_{j}(M(\Sigma))\right) t^{j} \\
& =\frac{1}{\left(1-t^{2}\right)\left(1-t^{4}\right)}\left(\left(1+t^{3}\right)^{2 g}-t^{2 g}(1+t)^{2 g}\right) .
\end{aligned}
$$

Notice that the coefficients of $t^{j}$ in the expansion of $(1+t)^{2 g}$ are the dimensions of the exterior powers $\Lambda^{j}$. Thus we have

$$
\sum_{j} \operatorname{dim}\left(H_{j}(M(\Sigma))\right) t^{j}=\frac{1}{\left(1-t^{2}\right)\left(1-t^{4}\right)} \sum \operatorname{dim}\left(\Lambda^{i}\right)\left(t^{3 i}-t^{2 g+i}\right) .
$$

This formula strongly suggests that there should be a natural (ie mapping class group equivariant) identification of the homology groups themselves, which we can write in the notation introduced above as

$$
\sum_{i} H_{i}(M(\Sigma)) t^{i}=\frac{1}{\left(1-t^{2}\right)\left(1-t^{4}\right)} \sum_{j} \Lambda^{j}\left(t^{3 j}-t^{2 g+j}\right),
$$


Analysis of the argument of Atiyah and Bott shows that this is indeed the case: the starting point is the fact that the homology of the moduli space injects into the homology of the space of all irreducible connections where the action of the mapping class group is clear. The formulae are again simpler if we use the reorganised grading $\Lambda_{(j)}$. Similarly, we write $V^{(C, i)}(\Sigma)$ for the homology group $H_{(3 g-3-i)}(M(\Sigma))$. Then we have

$$
\begin{aligned}
& \sum_{i} V^{(C, i)}(\Sigma) t^{i} \\
& \quad=t^{-(3 g-3)} \frac{1}{\left(1-t^{2}\right)\left(1-t^{4}\right)} \sum_{j \geq 0} \Lambda_{(j)}\left(t^{3 g+3 j}+t^{3 g-3 j}-t^{3 g+j}-t^{3 g-j}\right) \\
& \quad=\sum_{j>0} \frac{\left(t^{2 j}-t^{-2 j}\right)\left(t^{j}-t^{-j}\right)}{\left(t^{2}-t^{-2}\right)\left(t-t^{-1}\right)} \Lambda_{(j)}(\Sigma) .
\end{aligned}
$$

Writing

$\frac{\left(t^{2 j}-t^{-2 j}\right)\left(t^{j}-t^{-j}\right)}{\left(t^{2}-t^{-2}\right)\left(t-t^{-1}\right)}=\left(t^{2 j-2}+t^{2 j-4}+\cdots+t^{4-2 j}+t^{2-2 j}\right)\left(t^{j-1}+\cdots+t^{1-j}\right)$,

we can evaluate at $t=1$ to get a formula for the total homology

$$
V^{C}(\Sigma)=H_{*}(M(\Sigma))=\sum j^{2} \Lambda_{(j)} .
$$

\section{Scheme of proof}

We begin by bringing the Alexander polynomial into the picture, and for this we recall some multilinear algebra, which one can find in [10]. Suppose $U_{0}, U_{1}$ are finite-dimensional vector spaces and $\Gamma$ is a linear subspace of $U_{0} \oplus U_{1}$. Then, up to a scalar factor, $\Gamma$ defines a Plucker point $|\Gamma|$ in the exterior algebra $\Lambda^{*}\left(U_{0} \oplus U_{1}\right)$. In turn, up to a scalar ambiguity, elements of this exterior algebra can be viewed as linear maps from $\Lambda^{*}\left(U_{0}\right)$ to $\Lambda^{*}\left(U_{1}\right)$, so we have

$$
|\Gamma|: \Lambda^{*}\left(U_{0}\right) \rightarrow \Lambda^{*}\left(U_{1}\right)
$$

defined up to a scalar. More precisely, $|\Gamma|$ is defined once one chooses a trivialisation of the line $\Lambda^{\max }(\Gamma) \otimes \Lambda^{\max }\left(U_{0}\right)^{*}$. In the case when $\Gamma$ is the graph of a linear map $f: U_{0} \rightarrow U_{1}$ there is a natural trivialisation of this line and the map $|\Gamma|$ is the usual map $\Lambda^{*}(f)$ induced on the exterior powers. In general the construction satisfies a composition rule, for the case when one has another subspace $\Gamma^{\prime} \subset U_{1} \oplus U_{2}$, provided the subspaces satisfy a transversality condition. One wants the sum of the projection maps from $\Gamma \oplus \Gamma^{\prime}$ to $U_{1}$ to be surjective. Then if one defines $\Gamma^{\prime \prime} \subset U_{0} \oplus U_{2}$ to be set of pairs $\left(u_{0}, u_{2}\right)$ for which there 
exists a $u_{1} \in U_{1}$ with $\left(u_{0}, u_{1}\right) \in \Gamma,\left(u_{1}, u_{2}\right) \in \Gamma^{\prime}$, one has $\left|\Gamma^{\prime \prime}\right|=\left|\Gamma^{\prime}\right| \circ|\Gamma|$, if one uses an appropriate rule for normalising the scalar ambiguities. We apply these ideas in the case of a cobordism $W$ between surfaces $\Sigma_{0}, \Sigma_{1}$, of the kind considered above, so $H_{1}\left(\Sigma_{0}\right) \oplus H_{1}\left(\Sigma_{1}\right)$ generates $H_{1}(W)$. We let $U_{i}=H_{1}\left(\Sigma_{i}\right)$ and let $\Gamma \subset U_{0} \oplus U_{1}$ be the kernel of the inclusion map. Equivalently, by Poincaré duality, we can take $U_{i}=H^{1}\left(\Sigma_{i}\right)$ and let $\Gamma$ be the image of the restriction map, so under our hypotheses $\Gamma \cong H^{1}(W)$. We use the integer lattices in all these spaces to fix the scalar ambiguities, at least up to a sign, which we are not going to keep track of. The conclusion is that we get a linear map

$$
\alpha_{W}: \Lambda^{*}\left(H^{1}\left(\Sigma_{0}\right)\right) \rightarrow \Lambda^{*}\left(H^{1}\left(\Sigma_{1}\right)\right) .
$$

This can be regarded as a prototype of the general construction recalled in Section 2. Indeed we can obtain the map in a gauge theory framework by considering moduli spaces of flat $S^{1}$ connections: the exterior algebra then appears as the homology of the Jacobian torus $H^{1}(\Sigma ; \mathbf{R}) / H^{1}(\Sigma ; \mathbf{Z})$ parametrising flat connections over $\Sigma$. Now consider how the gradings work. In general if $U_{i}$ has dimension $n_{i}$ and $\Gamma$ has dimension $r$ the map $|\Gamma|$ increases degree by $n_{0}-r$. In our case, Poincaré duality implies that

$$
\operatorname{dim}(\Gamma)=\operatorname{dim}\left(H^{1}(W)\right)=\frac{1}{2}\left(\operatorname{dim} H^{1}\left(\Sigma_{0}\right)+\operatorname{dim} H^{1}\left(\Sigma_{1}\right)\right),
$$

and this means that $\alpha_{W}$ preserves the modified grading of the exterior powers, so we have

$$
\alpha_{i, W}: \Lambda_{(i)}\left(\Sigma_{0}\right) \rightarrow \Lambda_{(i)}\left(\Sigma_{1}\right) .
$$

As it stands, this notation is a little ambiguous because $\Lambda_{(i)}(\Sigma)$ is used to denote the two (isomorphic) exterior powers $\Lambda^{g \pm i}$, and we have maps induced on each one. Let $\omega_{i}$ be the intersection form on $H_{1}\left(\Sigma_{i}\right)$, and consider the symplectic form $\left(\omega_{0},-\omega_{1}\right)$ on the product. Then the subspace $\Gamma_{W}$ is a Lagrangian subspace of the product with this symplectic form - this is just the standard consequence of Poincaré-Lefschetz duality for $(W, \partial W)$. Then one can readily show that the two maps associated to such a Lagrangian subspace are equal.

This construction behaves well with respect to the composition of cobordisms. If $W^{\prime}$ is a cobordism from $\Sigma_{1}$ to $\Sigma_{2}$ and we form the composite cobordism $W^{\prime \prime}$ by gluing $W$ to $W^{\prime}$ along $\Sigma_{1}$ and if $W^{\prime \prime}$ satisfies our homological condition $H_{1}\left(W^{\prime \prime}, \partial W^{\prime \prime}\right)=\mathbf{Z}$ then the transversality condition holds at $\Sigma_{1}$ and the composite subspace $\Gamma^{\prime \prime}$ corresponds to $H^{1}\left(W^{\prime \prime}\right)$.

Now suppose that $\Sigma_{0}$ and $\Sigma_{1}$ are identified and we glue the ends of $W$ to obtain a closed manifold $\bar{W}$ as before. We also have a preferred element $\theta$ of $H^{1}(\bar{W})$, the Poincaré dual of the image of the boundary components, so we have an Alexander polynomial of the pair $(\bar{W}, \theta)$. 
Proposition 12 The Alexander polyomial of $(\bar{W}, \theta)$ is $a_{0}+\sum_{j} a_{j}\left(t^{j}+t^{-j}\right)$ where

$$
a_{j}=(-1)^{j} \operatorname{Trace}\left(\alpha_{j, W}: \Lambda_{(j)}\left(\Sigma_{0}\right) \rightarrow \Lambda_{(j)}\left(\Sigma_{0}\right)\right) .
$$

Let $\tilde{W}$ be the infinite cyclic cover of $\bar{W}$ corresponding to $\theta$. The Alexander polynomial is defined from the homology $H_{1}(\tilde{W})$, regarded as a module over the ring $\Lambda=\mathbf{Z}\left[t, t^{-1}\right]$ of Laurent series. Write $V_{\mathbf{Z}}$ for the homology group $H_{1}\left(\Sigma_{0}\right)$ with integral coefficients. We have a subgroup $\Gamma_{\mathbf{Z}} \subset V_{\mathbf{Z}} \times V_{\mathbf{Z}}$, the integer lattice in $\Gamma$, such that

$$
H_{1}(W ; \mathbf{Z})=\left(V_{\mathbf{Z}} \oplus V_{\mathbf{Z}}\right) / \Gamma_{Z} .
$$

Thinking of $\tilde{W}$ as constructed from a chain of copies of $W$, glued along the boundaries, we see that, as an abelian group

$$
H_{1}(\tilde{W})=\left(\ldots V_{\mathbf{Z}} \oplus V_{\mathbf{Z}} \oplus V_{\mathbf{Z}} \ldots\right) / \sim,
$$

where $\sim$ is the equivalence relation generated by

$$
(\ldots, 0,0, \sigma, 0,0, \ldots) \sim(\ldots, 0,0,0, \tau, 0, \ldots)
$$

if $(\sigma, \tau) \in \Gamma_{\mathbf{Z}}$. In other words

$$
H_{1}(\tilde{W})=\Lambda \otimes_{\mathbf{z}} V_{\mathbf{Z}} / \sim
$$

where $\lambda \otimes \sigma \sim t \lambda \otimes \tau$ for $(\sigma, \tau) \in \Gamma_{\mathbf{Z}}$. Explicitly, this says that we can construct a square presentation matrix for $H_{1}(\tilde{W})$ as a $\Lambda$-module in the following way. Let $\left(e_{j}\right)$ be a standard basis for $H_{1}\left(\Sigma_{0}\right)$ and let $\left(\gamma_{i}\right)$ be a basis for $\Gamma_{\mathbf{Z}}$. We can write $\gamma_{i}=\left(\sigma_{i}, \tau_{i}\right) \in V_{\mathbf{Z}} \times V_{\mathbf{Z}}$, and express these in terms of the basis:

$$
\sigma_{i}=\sum_{j} \sigma_{i j} e_{j}, \quad \tau_{i}=\sum_{j} \tau_{i j} e_{j}
$$

Then the $2 g$ elements $e_{i}$ can be thought of as generators of $H_{1}(\tilde{W})$ over $\Lambda$ with $2 g$ relations

$$
\sum_{j}\left(\sigma_{i j} e_{j}-t \tau_{i j} e_{j}\right)=0
$$

So the presentation matrix is just $\left(\sigma_{i j}-t \tau_{i j}\right)$. By definition the Alexander polynomial (up to a unit in $\Lambda$ ) is the determinant $\delta(t)=\operatorname{det}\left(\sigma_{i j}-t \tau_{i j}\right)$. It is now a straightforward exercise in exterior algebra to complete the proof of the proposition, up to a multiplication by a unit $\pm t^{\mu}$, just working through the definitions in the Plucker construction. Notice the particular case when $\Gamma$ is the graph of a linear map $f: V \rightarrow V$. In this case we can choose bases so that $\left(\sigma_{i j}\right)$ is the identity matrix, and $\tau_{i j}$ is the matrix of $f$ in the usual sense. The 
assertion that we want comes down to the familiar fact that the coefficients of the characteristic polynomial are $\pm \operatorname{Tr}\left(\Lambda^{k} f\right)$.

With regard to the ambiguity in the definition of the Alexander polynomial, recall that this is reduced to \pm 1 by specifying that $\Delta(t)= \pm t^{\mu} \delta(t)$ should have $\Delta(t)=\Delta\left(t^{-1}\right)$. So to complete the proof of Propostion 12, up to our overall \pm 1 ambiguity, we need only use the fact noted above that the traces of the $\alpha_{i, W}$ satisfy this symmetry property.

We see now that the two formulae, for closed 3-manifolds, can be deduced if one proves the obvious relative versions for cobordisms. Suppose we have a theory in which we have fixed isomorphisms, for all surfaces $\Sigma$,

$$
V(\Sigma)=\bigoplus_{j \geq 0} R_{j} \otimes \Lambda_{(j)}(\Sigma)
$$

where the $R_{i}$ are universal vector spaces, independent of $\Sigma$. Suppose we can prove that for all cobordisms $W$ from $\Sigma_{0}$ to $\Sigma_{1}$ the map $\rho_{W}: V\left(\Sigma_{0}\right) \rightarrow V\left(\Sigma_{1}\right)$ is equal (under these fixed isomorphisms) to a direct sum

$$
\rho_{W}=\bigoplus_{i} 1_{R_{j}} \otimes \alpha_{j, W}
$$

Then in the case when $\Sigma_{0}=\Sigma_{1}$ the gluing axioms and Proposition 12 imply that the numerical invariant of the closed manifold $\bar{W}$ is

$$
\sum_{j} \operatorname{dim}\left(R_{j}\right) a_{j}
$$

In particular it is then clear that the calculations of Section 3 will imply the Casson and Meng-Taubes formulae. Now this relative version is both more general and easier to prove. The gluing axioms in our topological field theory and the functorial property of the Plucker construction mean that if the statement is true for two composeable cobordisms it is also true for the composite. We can decompose any cobordism $W$ into a composite of elementary cobordisms and it suffices to prove the result for these. The homological condition (3) means that we can choose these elementary cobordisms to have index 1 or 2 ; that is, we reduce to considering the standard elementary cobordisms $Z$ from $\Sigma^{g}$ to $\Sigma^{g+1}$ and $Z^{\prime}$ from $\Sigma^{g+1}$ to $\Sigma^{g}$, where $\Sigma^{g}$ is a standard surface of genus $g$. To sum up then we have to establish, in the Seiberg-Witten and Casson theories, the existence of isomorphisms (13) and the property (14) for these elementary cobordisms. 


\section{$5 \quad$ Naturality arguments}

We will now see that the proof can be completed without any further geometric input, using algebra and topology. In a nutshell, we shall show that the invariants are determined by their formal properties. We recall some representation theory of the (real) symplectic group. The representations $\Lambda^{i}$ of $S p(2 g, \mathbf{R})$ are not in general irreducible. Let $L: \Lambda^{i} \rightarrow \Lambda^{i+2}$ be the wedge product with the symplectic form $\omega \in \Lambda^{2}$. The primitive subspace $P^{i}$ is the kernel of

$$
L^{n-i+1}: \Lambda^{i} \rightarrow \Lambda^{2 n-i+2} .
$$

The decomposition of the exterior powers into irreducible representations is, for $i \leq n$,

$$
\Lambda^{i}=P^{i} \oplus P^{i-2} \oplus \ldots,
$$

[6], where we use the maps $L^{j}$ to map $P^{i-2 j}$ into $\Lambda^{i}$. Thus

$$
P^{i}=\Lambda^{i}-\Lambda^{i-2},
$$

as virtual representations. We shall also need to use the fact that the $P^{i}$ are irreducible representations of the discrete subgroup $S p(2 n, \mathbf{Z}) \subset S p(2 n, \mathbf{R})$, and of the finite-index subgroups of $S p(2 n, \mathbf{Z})$ associated to spin structures. This refinement follows because these subgroups are Zariski-dense in $S p(2 n, \mathbf{R})$.

We now modify the grading of the primitive spaces, writing $P_{(i)}=P^{n-i}$, so

$$
\Lambda_{(i)}=P_{(i)}+P_{(i+2)}+\ldots,
$$

We associate to a surface $\Sigma$ vector spaces

$$
P_{(i)}(\Sigma)=P_{(i)}\left(H_{1}(\Sigma)\right)
$$

which give irreducible representations of the mapping class group. Now suppose we have a theory in which we know that

$$
V(\Sigma)=\sum \mu_{j} \Lambda_{(j)},
$$

as representations of the mapping class group, for certain multiplicities $\mu_{i}$. Suppose the theory is $\mathbf{Z} / \mathbf{2}$-graded, with the even/odd part made of the $\Lambda_{(\text {even) }}$, $\Lambda_{\text {(odd) }}$ respectively. Suppose the multiplicities $\mu_{j}$ are universal, independent of the genus. All of these conditions hold in our two geometric examples. Then we can write

$$
V(\Sigma)=\sum_{j} \nu_{j} P_{(j)},
$$

where

$$
\nu_{0}=\mu_{0}, \nu_{1}=\mu_{1}, \nu_{2}=\mu_{0}+\mu_{2}, \ldots
$$


Define vector spaces $Q_{j}$ for $0 \leq j \leq g(\Sigma)$ by

$$
Q_{j}(\Sigma)=\operatorname{Hom}_{M C(\Sigma)}\left(P_{(j)}(\Sigma), V(\Sigma)\right)
$$

where $\operatorname{Hom}_{M C(\Sigma)}$ denotes the equivariant homomorphisms under the action of the mapping class group. So the $Q_{j}$ are vector spaces canonically associated to a surface and by construction the mapping class group acts trivially on them. On the other hand we have canonical isomorphisms

$$
V(\Sigma)=\bigoplus_{j} Q_{j}(\Sigma) \otimes P_{(j)}(\Sigma)
$$

since the $P_{(j)}$ are distinct irreducible representations of $\operatorname{Sp}(2 n, \mathbf{R})$ and hence of the mapping class group. (Here we use the fact that the mapping class group maps onto $S p(2 n \mathbf{Z})$.) Notice that the dimensions of the $Q_{j}(\Sigma)$ are the integers $\nu_{j}$ determined by the $\mu_{j}$. and hence are universal, independent of the genus.

At this stage we bring in the following lemma, whose proof we leave as an exercise for the reader. (For our main proof we only need a special case of this - for the maps induced by elementary cobordisms - where the exercise is more straightforward.)

Lemma 19 Suppose $\left(U_{0}, \omega_{0}\right),\left(U_{1}, \omega_{1}\right)$ are symplectic vector spaces and $\Gamma \subset$ $U_{0} \times U_{1}$ is a Lagrangian subspace with respect to the symplectic form $\left(\omega_{0},-\omega_{1}\right)$ on $U_{0} \times U_{1}$. Then the Plucker map $|\Gamma|$ maps $P_{(j)}\left(U_{0}\right)$ to $P_{(j)}\left(U_{1}\right)$.

It follows that a cobordism $W$ defines maps

$$
\beta_{j, W}: P_{(j)}\left(\Sigma_{0}\right) \rightarrow P_{(j)}\left(\Sigma_{1}\right),
$$

since the corresponding subspace $\Gamma_{W} \subset H_{1}\left(\Sigma_{0}\right) \times H_{1}\left(\Sigma_{1}\right)=H_{1}(\partial W)$ is Lagrangian. Consider the standard cobordism $Z$ from $\Sigma^{g}$ to $\Sigma^{g+1}$. This defines

$$
\rho_{Z}: \bigoplus_{j} Q_{j}\left(\Sigma^{g}\right) \otimes P_{(j)}\left(\Sigma^{g}\right) \rightarrow \bigoplus_{j} Q_{j}\left(\Sigma^{g+1}\right) \otimes P_{(j)}\left(\Sigma^{g+1}\right),
$$

where we have used the identifications (18) on the two ends of the cobordism.

Lemma 22 There are linear maps $\rho_{j}: Q_{j}\left(\Sigma^{g}\right) \rightarrow Q_{j}\left(\Sigma^{g+1}\right)$ such that

$$
\rho_{Z}=\bigoplus_{j} \rho_{j} \otimes \beta_{j, Z}
$$

We may construct the elementary cobordism $Z$ in a canonical way starting with the surface $\Sigma^{g+1}$ and a non-separating embedded circle $\delta \subset \Sigma^{g+1}$. The homology group $H_{1}\left(\Sigma^{g}\right)$ can be identified with the quotient $[\delta]^{\perp} /[\delta]$ of the 
annihilator $[\delta]^{\perp} \subset H_{1}\left(\Sigma^{g+1}\right)$ of $[\delta] \in H_{1}\left(\Sigma^{g+1}\right)$ with respect to the intersection form. Any diffeomorphism of $\Sigma^{g+1}$ that fixes $\delta$ induces a diffeomorphism of $Z$. Let $G$ be the group of automorphisms of $H_{1}\left(\Sigma^{g+1} ; \mathbf{Z}\right)$ that preserve the intersection form and fix $[\delta]$. We get a natural action of $G$ on $H_{1}\left(\Sigma^{g}\right)=[\delta]^{\perp} / \delta$. Any element of $G$ can be lifted to a diffeomorphism of $\Sigma^{g+1}$, fixing $\delta$, and hence to a diffeomorphism of $Z$. It follows from the naturality properties of our theory that $\rho_{Z}$ intertwines the $G$-actions on the spaces $\bigoplus Q_{j} \otimes P_{(j)}$ at the two ends, and these actions are induced from the actions on the $P_{(j)}$ since the mapping class groups act trivially on the $Q_{j}$. Now Lemma 22 follows from the $\mathbf{Z} / 2$-grading in the theory and the next, purely algebraic, Lemma.

Lemma 23 If $j+j^{\prime}$ is even the only non-zero $G$-equivariant maps from $P_{(j)}\left(\Sigma^{g}\right)$ to $P_{\left(j^{\prime}\right)}\left(\Sigma^{g+1}\right)$ are multiples of $\beta_{j, Z}$, in the case when $j=j^{\prime}$.

To see this we first choose another class $[\epsilon] \in H_{1}\left(\Sigma^{g+1}\right)$ with $[\epsilon] \cdot[\delta]=1$. This choice defines an isomorphism

$$
H_{1}\left(\Sigma^{g+1}\right)=H_{1}\left(\Sigma^{g}\right) \oplus\langle[\epsilon],[\delta]\rangle,
$$

and hence a copy of $S p(2 g, \mathbf{Z})$ in $G$. It is easy to verify that we have then have natural (ie $S p(2 g, \mathbf{Z})$-invariant) isomorphisms

$$
P_{(j)}\left(\Sigma^{g+1}\right)=P_{(j+1)}\left(\Sigma^{g}\right) \oplus P_{(j)}\left(\Sigma^{g}\right) \otimes\left\langle[\epsilon, \delta] \oplus P_{(j-1)}\left(\Sigma^{g}\right) .\right.
$$

Using the fact that the $P_{(j)}$ are distinct irreducible representations we see that the only $S p(2 g, \mathbf{Z})$-equivariant maps are the maps in this decomposition from $P_{(j)}\left(\Sigma^{g}\right)$ to $P_{(j)}\left(\Sigma^{g}\right) \otimes\langle[\epsilon],[\delta]\rangle$ of the form $1 \otimes \lambda$, for $\lambda \in\langle[\delta],[\epsilon]\rangle$. But the only classes in $\langle[\delta],[\epsilon]\rangle$ which are fixed by the larger group $G$ are the multiples of $\delta$, so the only $G$-equivariant maps are multiples of $1 \otimes \delta$ which are just the multiples of $\beta_{j, Z}$.

Note that it follows from the definitions, and the trivial action on the $Q_{j}$, that the maps $\rho_{j}$ are universal in the following sense. If we start with surfaces $\Sigma^{g}, \Sigma^{g+1}$ and choose any standard cobordism between them we get the same maps $\rho_{j}$.

Lemma 24 The maps $\rho_{j}$ are isomorphisms, for $0 \leq j \leq g$.

We may carry through the entire discussion above for the other elementary cobordism $Z^{\prime}$ from $\Sigma^{g+1}$ to $\Sigma^{g}$. We get

$$
\rho_{Z^{\prime}}=\bigoplus \rho_{j}^{\prime} \otimes \beta_{j, Z^{\prime}},
$$

for universal maps $\rho_{j}^{\prime}$. Consider the trivial cobordism $\Sigma^{g} \times[0,1]$ and write this as a composite of elementary cobordisms $Z, Z^{\prime}$. That is, we introduce a pair 
of cancelling handles in the product. The map in our theory induced by the trivial cobordism is the identity map, and it follows that $\rho_{j}^{\prime} \circ \rho_{j}$ is the identity for each $j$. So the $\rho_{j}$ are injective. But we know, by our original hypothesis, that for $0 \leq j \leq g$

$$
\operatorname{dim} Q_{j}\left(\Sigma^{g}\right)=\operatorname{dim} Q_{j}\left(\Sigma^{g+1}\right),
$$

so the $\rho_{j}$ are isomorphisms, with inverses $\rho_{j}^{\prime}$.

Now we use the universal maps $\rho_{j}$ to identify the spaces $Q_{j}(\Sigma)$ for all surfaces $\Sigma$ (of genus $g \geq j$ ), and hence define universal vector spaces $Q_{j}$. The point is that this is unambigous since the mapping class groups acts trivially on the $Q_{j}(\Sigma)$ so it does not matter which cobordisms we use to induce the identifications. Then we have fixed isomorphisms

$$
V(\Sigma)=\bigoplus_{j} Q_{j} \otimes P_{(j)}(\Sigma)
$$

which, by construction, commute with the maps induced by elementary cobordisms, and hence by all cobordisms. Finally we can get back to the exterior powers, although this is not really necessary, since we can go straight from the primitive spaces to the Alexander polynomial. We know that the dimension of $Q_{j}$ is $\mu_{j}+\mu_{j-2}+\ldots$, so we may choose arbitrary embeddings

$$
\begin{aligned}
& Q_{0} \hookrightarrow Q_{2} \hookrightarrow Q_{4} \ldots \\
& Q_{1} \hookrightarrow Q_{3} \hookrightarrow Q_{5} \ldots
\end{aligned}
$$

and a sequence of complementary subspaces

$$
Q_{i}=Q_{i-2} \oplus R_{i}
$$

so that

$$
Q_{i}=R_{i} \oplus R_{i-2} \oplus R_{i-4} \ldots,
$$

and $\operatorname{dim} R_{i}=\mu_{i}$. Then we have fixed isomorphisms $V(\Sigma)=\bigoplus R_{j} \otimes \Lambda_{(j)}(\Sigma)$, which commute with the maps in the theory, as required.

\section{Connections with work of Thaddeus}

The main point of this article has been the fact that the homology of the flat connection moduli spaces, and of the symmetric products, can be described in terms of the exterior powers. It is natural to look for relations between these different geometric problems. In his paper [12], Thaddeus used an algebro-geometric construction to relate these objects - regarding the moduli space $M(\Sigma)$ as a moduli space of rank 2 holomorphic vector bundles. His idea is to study a 
moduli space of "stable pairs" $(E, \phi)$, where $E$ is a bundle and $\phi$ is a holomorphic section of $E$. The notion of stability depends on a real parameter $\tau \in(0, \infty)$, so there is a family of moduli spaces $\mathcal{M}_{\tau}$. When $\tau$ is small this fibres over the moduli space of vector bundles with fibre a complex projective space, and when $\tau$ is large $\mathcal{M}_{\tau}$ is simply a projective space. In between there are a finite number of exceptional values of $\tau$ where $\mathcal{M}_{\tau}$ changes by a "flip" (or "complex surgery") which can be described in terms of the symmetric products. So far in this paper we have avoided the case $d=0$ in the Seiberg-Witten theory. In this case there are reducible solutions of the equations over a surface, which causes some complications. However one can make a perturbation of the equation which removes the reducible solutions, and the relevant moduli space is then the symmetric product Sym ${ }^{g-1}(\Sigma)$. Thus we can extend our theory with vector spaces

$$
V_{0}=\Lambda_{(1)} \oplus 2 \Lambda_{(2)} \oplus \ldots
$$

Similarly, if we use perturbations, we obtain a theory for negative values of $d$ which brings in the higher symmetric products. (This is not the same as the unperturbed theory, in which there is a symmetry between $d$ and $-d$.) Thus

$$
V_{-d}=\Lambda_{-(d-1)} \oplus 2 \Lambda_{-(d-2)} \oplus \ldots,
$$

but using the symmetry $\Lambda_{(k)}=\Lambda_{(-k)}$ we get:

$$
V_{-d}=V_{d} \oplus d T
$$

where

$$
T=\Lambda_{0} \oplus 2 \Lambda_{1} \oplus \Lambda_{2} \ldots .
$$

(This corresponds to the homology of the Jacobian torus.) Now Thaddeus' relation between the homology of the different spaces can be written

$$
(2 g+1) H_{*}(M(\Sigma))=\sum_{j=0}^{2 g-1}(5 g-2-3 j) H_{*}\left(\operatorname{Sym}^{j}(\Sigma)\right)=\sum_{k=-d}^{k=d-1}(2 g+1+3 d) V_{d} .
$$

Using (25), the last sum yields the relation

$$
V^{C}=V_{0}+2 V_{1}+2 V_{2}+2 V_{3}+\ldots,
$$

which we can of course obtain equally well directly from (5), (9). This can be seen as a universal formula, relating the $(2+1)$-dimensional Casson theory and the $(2+1)$-dimensional Seiberg-Witten theory. These are both reductions of $(3+1)$ dimensional theories - the instanton theory in the Casson case - so the ideas should have some bearing on the problem of understanding the relation between these latter theories. Of course there are obvious similarities between Thaddeus' technique and those used in the programme of Pidstragatch and Tyurin in 4-dimensions. It would be interesting to obtain the relation between the theories directly using the Pidstragatch and Tyurin method. 


\section{References}

[1] S Akbulut, J Mc Carthy, Casson's Invariant for homology 3-spheres, Princeton. U.P. (1990)

[2] MF Atiyah, Topological Quantum Field Theory, Math. Publ. I.H.E.S. 68 (1988) 175-186

[3] M F Atiyah, R Bott, The Yang-Mills equations over Riemann surfaces, Phil. Trans. Roy. Soc. London, (1982) 308 523-615

[4] A Floer, An exact sequence for instanton homology, from: "The Floer Memorial Volume", Birkhauser (1995)

[5] C Frohman, A Nicas, An intersection-homology invariant for knots in rational homology 3-spheres, Topology, 33 (1993) 123-58

[6] P Griffiths, J Harris, Principles of Algebraic Geometry, John Wiley (1978)

[7] M Hutchins, Y Lee, Circle-valued Morse Theory, Reidemeister Torsion and the Seiberg-Witten invariant for 3-manifolds, Harvard University Preprint (1996)

[8] P Kronheimer, T Mrowka, The genus of embedded surfaces in the projective plane, Math. Res. Letters 1 (1994) 797-808

[9] G Meng, C H Taubes, $\underline{S W}=$ Milnor Torsion, Math. Res. Letters, 3 (1996) 661-674

[10] A Pressley, G B Segal, Loop Groups, Oxford U.P. (1980)

[11] C H Taubes, Casson's Invariant and Gauge Theory, Jour. Differential Geometry, 25 (1990) 547-599

[12] M Thaddeus, Stable pairs, linear systems and the Verlinde formula, Inv. Math. 117 (1994) 317-53

Department of Mathematics

Imperial College, London SW7 2BZ, UK

Email: s.donaldson@ic.ac.uk

Received: 5 March 1999 Revised: 24 June 1999 